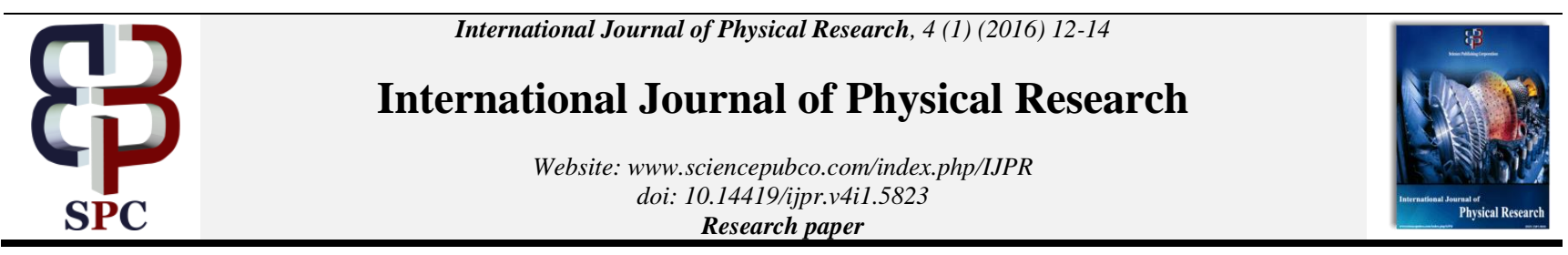

\title{
Soliton solutions of nonlinear wave equation in finite de-formation elastic cylindrical rod by solitary wave ansatz method
}

\author{
S. Subhaschandra Singh * \\ Imphal College, Imphal- 795 001, Manipur, India \\ *Corresponding author E-mail: subhasic@yahoo.co.in
}

\begin{abstract}
In this paper, we consider nonlinear wave equation in finite deformation elastic cylindrical rod and obtain soliton solutions by Solitary Wave Ansatz method. It is shown that the ansatz method provides a very effective and powerful mathematical tool for obtaining solutions for Nonlinear Evolution Equations (NLEEs) in nonlinear Science.
\end{abstract}

Keywords: Elastic Rod; Finite Deformation; Nonlinear Wave Equation; Solitary Wave Ansatz Method; Soliton.

\section{Introduction}

In the recent a few decades, solid mechanics had been taking more and more account of nonlinear effects and had acquired considerable development in the studies of Solitons. Many researchers had given attention on the propagation of longitudinal waves in a nonlinear elastic cylindrical rod. [1- 3]. When a compression wave propagates longitudinally along a rod, the Poisson effect causes a lateral motion simultaneously. Such a lateral motion produces contribution to the kinetic energy of the system and also to the strain energy as well. Many methods have been proposed so far for obtaining solutions of Nonlinear Evolution Equations (NLEEs). In this paper, longitudinal oscillations of a nonlinear elastic cylindrical rod are studied. The nonlinear longitudinal wave equation of the elastic rod derived by Liu and Zhang [5, 6]] is solved by the Solitary Wave Ansatz Method [4].

The nonlinear wave equation in a finite deformation elastic cylindrical rod reads

$$
\frac{\partial^{2} \mathrm{u}}{\partial \mathrm{t}^{2}}-\mathrm{c}_{0}{ }^{2} \frac{\partial^{2} \mathrm{u}}{\partial \mathrm{x}^{2}}=\frac{1}{2} \frac{\partial}{\partial \mathrm{x}}\left[\begin{array}{c}
3 \mathrm{c}_{0}{ }^{2}\left(\frac{\partial \mathrm{u}}{\partial \mathrm{x}}\right)^{2}+\mathrm{c}_{0}{ }^{2}\left(\frac{\partial \mathrm{u}}{\partial \mathrm{x}}\right)^{3} \\
+\sigma^{2} \mathrm{R}^{2} \frac{\partial}{\partial \mathrm{x}}\left(\frac{\partial^{2} \mathrm{u}}{\partial \mathrm{t}^{2}}\right)-\mathrm{c}_{\mathrm{s}}{ }^{2} \sigma^{2} \mathrm{R}^{2} \frac{\partial^{3} \mathrm{u}}{\partial \mathrm{x}^{3}}
\end{array}\right],
$$

where $u(x, t)$ is an unknown displacement, $c_{0}=\sqrt{E / \rho}$ is the speed of the longitudinal wave in the rod, $c_{s}=\sqrt{\mu / \rho}$ is the speed of the shear wave in an unbounded medium, $\mathrm{E}$ is the Young's modulus, $\rho$ is the density, $\mu$ is the shear modulus, $\sigma$ is the Poisson ratio and $\mathrm{R}$ is the radius of the rod. It is known that when the longitudinal wave propagates, the lateral shear wave caused by transverse Poisson effect also propagates.

Differentiating both sides of eqn. (1) with respect to $\mathrm{x}$ and putting $\psi=\frac{\partial \mathrm{u}}{\partial \mathrm{x}}$, we write,

$$
\frac{\partial^{2} \psi}{\partial \mathrm{t}^{2}}-\mathrm{c}_{0}{ }^{2} \frac{\partial^{2} \Psi}{\partial \mathrm{x}^{2}}=\frac{1}{2} \frac{\partial^{2}}{\partial \mathrm{x}^{2}}\left[\begin{array}{l}
3 \mathrm{c}_{0}{ }^{2} \psi^{2}+\mathrm{c}_{0}{ }^{2} \psi^{3}+ \\
\sigma^{2} \mathrm{R}^{2}\left(\frac{\partial^{2} \Psi}{\partial \mathrm{t}^{2}}-\mathrm{c}_{\mathrm{S}}{ }^{2} \frac{\partial^{2} \psi}{\partial \mathrm{x}^{2}}\right)
\end{array}\right]
$$

2. Reduced Nonlinear Ordinary Differential Equation (RNLODE): Let $\psi(\mathrm{x}, \mathrm{t})$ be a one-dimensional travelling wave solution for a nonlinear partial differential equation (NLPDE) of the form

$\mathrm{P}\left(\psi, \psi_{\mathrm{x}}, \psi_{\mathrm{t}}, \psi_{\mathrm{xx}}, \psi_{\mathrm{tt}}, \psi_{\mathrm{xxt}}, \psi_{\mathrm{xtt}} \ldots\right)=0$.

where

$\psi_{\mathrm{x}}=\frac{\partial \psi}{\partial \mathrm{x}}$, etc.

Let us introduce a new dimensionless variable

$\xi=\mathrm{kx}-\omega \mathrm{t}+\varphi$,

where $\mathrm{k}$ is the wave number, $\omega$ is the circular frequency and $\varphi$ is the phase constant of the travelling wave. Then, we have,

$\psi(\mathrm{x}, \mathrm{t})=\psi(\xi)$.

Also, $\frac{\partial}{\partial \mathrm{t}} \equiv-\omega \frac{\mathrm{d}}{\mathrm{d} \xi}, \frac{\partial}{\partial \mathrm{x}} \equiv \mathrm{k} \frac{\mathrm{d}}{\mathrm{d} \xi}$,

$\frac{\partial^{2}}{\partial \mathrm{t}^{2}} \equiv \omega^{2} \frac{\mathrm{d}^{2}}{\mathrm{~d} \xi^{2}}, \frac{\partial^{2}}{\partial \mathrm{x}^{2}} \equiv \mathrm{k}^{2} \frac{\mathrm{d}^{2}}{\mathrm{~d} \xi^{2}}$,

$\frac{\partial^{2}}{\partial \mathrm{x} \partial \mathrm{t}} \equiv-\mathrm{k} \omega \frac{\mathrm{d}^{2}}{\mathrm{~d} \xi^{2}}$, etc.

Using eqns. (5) and (6), eqn. (3) reduces to a RNLODE of the form

$\mathrm{Q}\left(\psi, \psi^{\prime}, \psi^{\prime \prime}, \psi^{\prime \prime \prime}, \ldots\right)=0$

where 
$\psi^{\prime}=\frac{\mathrm{d} \psi}{\mathrm{d} \xi}, \psi^{\prime \prime}=\frac{\mathrm{d}^{2} \psi}{\mathrm{d} \xi^{2}}$, etc.

Solving RNLODE (7), we can obtain the solution of NLEE (3) as well.

For the present problem, using eqns. (5) and (6), eqn. (2) reduces to a nonlinear ordinary differential equation

$$
\begin{aligned}
& \left(\omega^{2}-c_{0}{ }^{2} k^{2}\right) \frac{d^{2} \psi}{d \xi^{2}} \\
& =k^{2} \frac{d^{2}}{d \xi^{2}}\left[\begin{array}{c}
\frac{3}{2} c_{0}{ }^{2} \psi^{2}+\frac{c_{0}{ }^{2}}{2} \psi^{3} \\
+\frac{\sigma^{2} R^{2}}{2}\left(\omega^{2}-c_{s}{ }^{2} k^{2}\right) \frac{d^{2} \psi}{d \xi^{2}}
\end{array}\right]
\end{aligned}
$$

Integrating eqn. (8) with respect to $\xi$ twice and choosing the integration constants as zero, we have,

$$
\begin{aligned}
& \left(\omega^{2}-\mathrm{c}_{0}{ }^{2} \mathrm{k}^{2}\right) \psi=\frac{3}{2} \mathrm{c}_{0}{ }^{2} \mathrm{k}^{2} \psi^{2}+\frac{\mathrm{c}_{0}{ }^{2} \mathrm{k}^{2}}{2} \psi^{3}+\frac{\sigma^{2} \mathrm{R}^{2} \mathrm{k}^{2}}{2}\left(\omega^{2}-\right. \\
& \left.\mathrm{c}_{\mathrm{s}}{ }^{2} \mathrm{k}^{2}\right) \frac{\mathrm{d}^{2} \psi}{\mathrm{d} \xi^{2}}
\end{aligned}
$$

Dividing both sides of the above eqn. by

$\left(\omega^{2}-c_{s}{ }^{2} k^{2}\right) \frac{\sigma^{2} R^{2} k^{2}}{2}$, we obtain,

$\frac{d^{2} \psi}{d \xi^{2}}-\beta_{1} \psi+\beta_{2} \psi^{2}+\beta_{3} \psi^{3}=0$

where use has been made of

$\beta_{1}=-\frac{2\left(\omega^{2}-\mathrm{c}_{0}^{2} \mathrm{k}^{2}\right)}{\left(\omega^{2}-\mathrm{c}_{\mathrm{s}}^{2} \mathrm{k}^{2}\right) \sigma^{2} \mathrm{R}^{2} \mathrm{k}^{2}}$

$\beta_{2}=\frac{3 c_{0}^{2}}{\left(\omega^{2}-c_{s}^{2} k^{2}\right) \sigma^{2} R^{2}}$

$\beta_{3}=\frac{\mathrm{c}_{0}{ }^{2}}{\left(\omega^{2}-\mathrm{c}_{\mathrm{s}}{ }^{2} \mathrm{k}^{2}\right) \sigma^{2} \mathrm{R}^{2}}$

\section{Application of solitary wave ansatz method}

In this section, the solitary wave ansatz method is used to obtain the soliton solution of the nonlinear wave equation in finite deformation elastic rod.

Let us assume the following form of ansatz solution

$\psi(\xi)=A+B \operatorname{sech}^{p}(\mu \xi), p>0$

where $\xi=\mathrm{kx}-\omega \mathrm{t}+\varphi$; $\mathrm{A}, \mathrm{B}$ are constants, $\mu$ is the inverse width of the soliton and $\varphi$ is a phase constant. The index $p$ is to be calculated latter.

We have,

$\frac{d^{2} \psi}{d \xi^{2}}=p^{2} \mu^{2} B \operatorname{sech}^{p} \mu \xi$

$-p(p+1) \mu^{2} B \operatorname{sech}^{p+2} \mu \xi$.

Substituting eqns. (11) and (12) into eqn. (9), we obtain,

$p^{2} \mu^{2} B \operatorname{sech}^{p} \mu-p(p+1) \mu^{2} B \operatorname{sech}^{p+2} \mu \xi$

$+\beta_{1}\left(A+B \operatorname{sech}^{p} \mu \xi\right)$

$+\beta_{2}\left(A^{2}+B^{2} \operatorname{sech}^{2 p} \mu \xi+2 A B \operatorname{sech}^{p} \mu \xi\right)$

$+\beta_{3}\left(\begin{array}{c}A^{3}+B^{3} \operatorname{sech}^{3 p} \mu \xi+3 A^{2} B \operatorname{sech}^{p} \mu \xi \\ +3 A B^{2} \operatorname{sech}^{2 p} \mu \xi\end{array}\right)=0$

Equating the exponents $p+2$ and $3 p$ in eqn (13), we obtain $\mathrm{p}=1$.

Equating the co-efficients of the linearly independent functions $\operatorname{sech}^{m p} \mu \xi(m=0,1,2,3)$ successively to zero and using eqn (14), we obtain,

$\beta_{1}+\beta_{2} A+\beta_{3} A^{2}=0$,

$\mu^{2}+\beta_{1}+2 \beta_{2} A+3 \beta_{3} A^{2}=0$,

$\beta_{2}+3 \beta_{3} A=0$

$-2 \mu^{2}+\beta_{3} B^{2}=0$.

Using eqns. (10) and (15), we obtain,

$A=-\frac{\beta_{2}}{3 \beta_{3}}=-1$,

$\mu=\sqrt{\frac{\left(2 \omega^{2}+\mathrm{c}_{0}^{2} \mathrm{k}^{2}\right)}{\left(\omega^{2}-\mathrm{c}_{\mathrm{s}}^{2} \mathrm{k}^{2}\right) \sigma^{2} \mathrm{R}^{2} \mathrm{k}^{2}}}$

[considering only the positive value),

$\mathrm{B}= \pm \sqrt{\frac{2\left(2 \omega^{2}+\mathrm{c}_{0}^{2} \mathrm{k}^{2}\right)}{\mathrm{c}_{0}^{2}}}$

Now, the solution of eqn. (9) is obtained as

$\psi(\xi)=-1$

$\pm\left[\sqrt{\frac{2\left(2 \omega^{2}+\mathrm{c}_{0}^{2} \mathrm{k}^{2}\right)}{\mathrm{c}_{0}^{2}}}\right]$

$\times \operatorname{sech}\left[\left\{\sqrt{\left.\left.\frac{\left(2 \omega^{2}+\mathrm{c}_{0}^{2} \mathrm{k}^{2}\right)}{\left(\omega^{2}-\mathrm{c}_{\mathrm{s}}{ }^{2} \mathrm{k}^{2}\right) \sigma^{2} \mathrm{R}^{2} \mathrm{k}^{2}}\right\} \xi\right]}\right.\right.$

which is valid for $\omega>c_{s}$.

Since $\psi=\frac{d u}{d \xi}$, we obtain,

$\mathrm{u}(\xi)=\int \psi \mathrm{d} \xi+$ const.

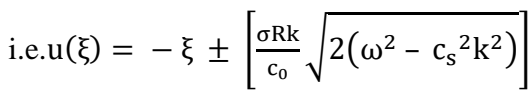

$\times \tan ^{-1}\left[\sinh \left\{\frac{\left(2 \omega^{2}+\mathrm{c}_{0}^{2} \mathrm{k}^{2}\right) \xi^{2}}{\left(\omega^{2}-\mathrm{c}_{\mathrm{s}}^{2} \mathrm{k}^{2}\right) \sigma^{2} \mathrm{R}^{2} \mathrm{k}^{2}}\right\}^{\frac{1}{2}}\right]$

Or,

$\mathrm{u}(\mathrm{x}, \mathrm{t})=-(\mathrm{kx}-\omega \mathrm{t}+\varphi)$

$\pm\left[\frac{\sigma R k}{c_{0}} \sqrt{2\left(\omega^{2}-c_{s}^{2} k^{2}\right)}\right]$

$\times \tan ^{-1}\left[\sinh \left\{\frac{\left(2 \omega^{2}+\mathrm{c}_{0}{ }^{2} \mathrm{k}^{2}\right)(\mathrm{kx}-\omega \mathrm{t}+\varphi)^{2}}{\left(\omega^{2}-\mathrm{c}_{\mathrm{s}}{ }^{2} \mathrm{k}^{2}\right) \sigma^{2} \mathrm{R}^{2} \mathrm{k}^{2}}\right\}^{\frac{1}{2}}\right]$

Thus, eqn (18) is the required solution of the problem.

\section{Conclusion}

In this paper, the nonlinear wave equation in finite deformation elastic rod has been solved by the solitary wave ansatz method. It is seen that the ansatz method is an efficient method for obtaining soliton solutions of many nonlinear evolution equations and the results of these will be useful in conducting research in future. 


\section{References}

[1] Z.F. Liu and S. Y. Zhang, SOLITARY WAVES IN FINITE DEFORMATION ELASTIC CIRCULAR ROD, Appl. Math. Mech.-Engl. Ed., 27 (10) (2006) 1431 - 1437.

[2] S. Y. Zhang and Z. F. Liu, Three kinds of nonlinear dispersive waves in elastic rods with finite deformation, Appl. Math. Mech. Engl. Ed. 29 (7) (2008) $909-917$.

[3] Guo Peng, Zhang Lei, Lu Ke-pu and Duan Wen-Shan, Solutions of nonlinear wave equation of elastic rod, Appl. Math. Mech. -Engl. Ed., 29 (1) (2008) 61-66.

[4] Peng Guo, Guixin Wan, Xiao Yun Wang and Xiao Wei Sun, New Soliton and Periodic Solutions for Nonlinear Wave Equation in Finite Deformation Elastic Rod, Int. J. Nonlinear Science 15 (2) (2013) 182- 192

[5] Marwan Alquran, Bright and Dark Soliton Solutions to the Ostrovsky Benjamin - Bona - Mahony (OS - BBM) Equation, J. Math. Com put. Sci. 2 (1) (2012) $15-22$.

[6] K.S. Ghafri, Analytic solutions of the Thomas equation by generalized tanh and travelling wave hypothesis methods, IJAMR 2 (2) (2013) $274-278$. 